\title{
The need for a focus on air pollution research in the elderly
}

\author{
T. Sandström*, A.J. Frew*, M. Svartengren ${ }^{\mp}$, G. Viegi ${ }^{+}$
}

The need for a focus on air pollution research in the elderly. T. Sandström, A.J. Frew, M. Svartengren, G. Viegi. (C) ERS Journals Ltd 2003.

ABSTRACT: During recent years an increasing focus has been directed towards the adverse health effects associated with ambient air pollution. Elderly people appear to be particularly susceptible to the adverse effects involving the respiratory and cardiovascular systems, resulting in symptoms, exacerbations of disease and even mortality.

From an epidemiological point of view it is essential to obtain a more detailed description and identification of factors associated with these health effects. Novel study designs are needed with complementary exposure and biomedical characterisation. Long-term prospective studies are required. A better understanding of the pathophysiological mechanisms is considered important and requires an interaction between epidemiological and mechanistic studies in elderly individuals with or without complementary diseases that put in them especially at risk.

Generally a synergy between complementary disciplines is warranted to move this important research area forward, also including in vitro models of cell responses in the elderly, animal models of diseases of the elderly, together with controlled air pollution exposure studies identifying health-related events and mechanisms.

The generation of an understanding of air pollution effects in the elderly, at an elevated level, is a prerequisite to substantially reducing the adverse health effects of this population group. At local, national and European Union levels, some steps have been taken to support the research in this area. A major focus on the adverse air pollution effects in the elderly requires a long-term commitment that still remains to be established.

Eur Respir J 2003; 21: Suppl. 40, 92s-95s.
*Dept of Respiratory Medicine and Allergy, University Hospital, Umeå, Sweden, ${ }^{\#}$ Respiratory Cell and Molecular Biology Research Division, Southampton General Hospital, Southampton, UK, "Karolinska Institute, Dept of Public Health Sciences, Division of Occupational Medicine, Stockholm, Sweden, ${ }^{+}$Pulmonary Environmental Epidemiology Group, CNR, Institute of Clinical Physiology, Pisa, Italy.

Correspondence: T. Sandström, Dept of Respiratory Medicine and Allergy, University Hospital, S-901 85 Umeå, Sweden.

Fax: 4690141369

E-mail: thomas.sandstrom@lung.umu.se

Keywords: Air pollution epidemiology controlled exposure studies

elderly

pathophysiological mechanisms

susceptibility

Received and accepted: April 122002
During recent years there has been an increasing focus on the adverse health effects of air pollutants which appear to affect all ages, from infants to the elderly [1-5]. Adverse health outcomes have mainly involved respiratory and cardiovascular systems and range from symptom changes to exacerbations of disease and even mortality. In parallel there has been increasing attention on the worldwide trend of increased respiratory morbidity in terms of asthma and chronic obstructive pulmonary disease (COPD) [6, 7]. COPD is projected to be the third leading cause of mortality and fifth leading cause of disability worldwide by the year 2020 .

The most obvious and appalling messages from epidemiological studies have been the increased mortality associated with air pollution events. The classical episodes of the Meuse valley 1930, Donora, Pennsylvania 1948, Pozo Rico, Mexico 1950, and London 1952 showed a clear escalation of death rates within the next few days after the onset of the smog. It is not currently known whether the lag time, often 2-3 days, was due to a single biological event or to a combination of different inflammatory and reactive mechanisms and events, overlapping for several days. This latter possibility could explain the spread of lag times from 1-7 days reported from different epidemiological studies. It is acknowledged that with current modelling techniques it is difficult to delineate the different biological events resulting in excess mortality and morbidity.

One of the major questions of the "Six cities study" [8] and subsequent studies showing an association of air pollution with mortality, has been whether the adjusted mortality rate ratios were really reflecting a true excess in mortality or, as some criticism suggested, merely reflected "harvesting" of people who would have died shortly afterwards, even if there had not been a pollution episode. Complementary studies have more recently demonstrated harvesting-resistant estimates of ambient air pollution on mortality, for instance from Philadelphia 1974-1988 [9].

Generally the epidemiological studies have shown airpollution-adjusted mortality-rate ratios, which are much higher for cardiovascular and respiratory disease as compared with other causes of death. This outcome has usually been higher in current smokers compared to exsmokers and other groups. Correlations have generally been strongest for death certificate diagnoses of heart failure and thrombotic disease, but are also strong for deaths from COPD [10, 11].

Considering the excess morbidity and mortality associated with air pollution observed in the epidemiological studies, there is considerable value in directing research at the underlying mechanisms. Possibilities for underlying mechanisms include: 1) excess activity of mechanism(s) that operate both in young and elderly people; 2) excessive impact of mechanism(s) that operate in young people but affects elderly people more, due to their decline in physical status; 3) mechanism(s) that only operate in the elderly; 4) Mechanism(s) that only operate in elderly people who are diseased.

Paediatricians are fond of saying that "children are not small adults". In a similar way, it could be said of elderly people: "they are not just adults who have lived longer". Not only have they been exposed to environmental factors much longer than younger people, but in addition the environment that they have been exposed to has, in many aspects, been 
Table 1.-Factors influencing air pollution responses in the elderly

Smoking history

Occupational exposure history

Environmental exposure history

Infections history

Altered immune response with age

Impaired homeostasis

Altered deposition of PM in diseased airways

Antioxidant and nutritional status

Respiratory, cardiovascular and other concurrent diseases Medication

PM: particulate matter.

quite different compared with the environment younger people are exposed to today. A number of aspects influencing the response of the elderly to air pollution are given in table 1 .

The levels of gaseous and particulate matter (PM) pollutants may have been quantitatively different during preceding decades. Since combustion was less well controlled and there may have been little or no measurement of pollutants, concentrations would often have been high for periods of time. The smog periods that repeatedly plagued many cities over the world in the past demonstrates that the exposures of individuals were considerably higher than today. These events may have resulted in airway and systemic responses in individuals, which may affect their sensitivity and responsiveness even today.

Qualitative physicochemical characteristics of PM pollutants may have been very different in the past, and the elderly may carry the effects of earlier exposures in life with them. Also it is important to consider past occupational exposure to a variety of different noxious agents both in outdoor and indoor environments. It has been proposed that extremely high levels of gaseous and particulate pollutants in the past have not been recognised sufficiently, but may for obvious reasons be difficult to include in the evaluation in epidemiological studies.

An aspect often overlooked is the impact of infections occurring many decades ago when the microbiological and the antibiotic situation was very different compared with today. Severe and sometimes persisting infections could not be treated due to lack of effective antibiotics. This often left persistent changes in a variety of organs including the cardiovascular system and the lungs, which may potentially translate into an enhanced susceptibility at old age. Prior infection may also affect local or systemic immune responses, which could result in an altered clinical response to air pollutants. Elderly individuals have been reported to show less marked acute phase responses, reduced cytotoxic cell activity, increased apoptosis and less systemic responses compared to younger people [12, 13]. Paradoxically older people may get less sick but be more likely to die, because of a contribution of physical fragility and less appropriate immune responses. Consequently, it is likely that immune and inflammatory responses to air pollutants may be less appropriate to protect the elderly individual, especially if already diseased. Additionally with age there is a general inability to keep homeostasis for different organ and systemic functions. Inappropriate heart rhythm variability has been suggested be one such malfunctions, which may result in circulatory instability and even death.

Interestingly, there are data suggesting that elderly individuals experience a higher deposition rate of PM in their airways, due to age-related changes in peripheral airway ventilation. In this respect COPD individuals have been proposed to have a substantially higher degree of particle deposition due to their peripheral airway obstruction and extremely uneven airways ventilation [14]. This means that these individuals may accommodate higher amounts of PM in the airways which potentially may enhance the adverse biological reactions elicited.

Other aspects of sensitivity to air pollutants, which may differ between younger and elderly individuals, are dietary habits leading to deficits of key dietary factors such as antioxidants, which have been found to be a part of the first line of defence to oxidative air pollutants [15]. Without a proper antioxidant protection more toxic secondary and tertiary products are produced in the epithelial lining fluid and cells in the airways. Comorbidities may affect the immune system, as well as a variety of medications taken by the elderly. Furthermore response to viral or bacterial infections could potentially be modulated by PM and gaseous air pollutants.

Of particular interest is the individual air pollution exposure profile in elderly individuals, who may spend little, if any time outdoors. Depending upon climatological aspects, open windows may alter the access of pollutants into homes. Still, even with closed windows and doors, the principal determinant of indoor particulate concentrations is the outdoor ambient particle level. Variations in outdoor particulate matter air pollution closely parallel variations in outdoor particle concentration. It must be recognised that there is currently relatively limited material that clarify the actual exposure situation of elderly individuals, particularly those that could be expected to be at risk for adverse air pollution-associated events.

\section{Focus for future research}

\section{Epidemiological studies}

On an individual basis there is an increasing need for measurements of complementary parameters (as yet to be clarified), which may relate to morbidity and mortality aspects. Of interest are studies of acute and chronic effects modulated by the presence of symptoms, like chronic mucus hypersecretion, degree and distribution of obstructive lung disease, respiratory infections, cardiovascular morbidity, as well as the presence of other environmental risk factors like active and passive smoking [16-18].

Time series analysis studies including complementary parameters are expected to continue to play a role in the future, but there is a definite lack of longitudinal surveys in the elderly [19-21]. Other aspects, which deserve further research, include questionnaires, lung function performance, complementary biological parameters, study design and interpretation in the elderly [18, 22].

Since the development of chronic effects cannot be estimated from short-term studies [23] prospective long-term studies are required. Not only would they need to contain enhanced environmental exposure assessment on an individual basis, but could also include a far more detailed characterisation of the clinical and physiological status of individuals. Such surveys could also be performed in association with laboratories measuring relevant biomarkers of exposures, as well as early and chronic health effects. In this respect, a complementary understanding of relevant biomarkers may be obtained from scientists working with human experimental exposure systems evaluating effects in elderly individuals with various pre-existing respiratory and cardiovascular diseases, in comparison to healthy elderly and younger subjects [24, 25]. A thoroughly designed longitudinal survey of air pollution epidemiology can represent a biological extension of the environmental impact assessment (fig. 1). 


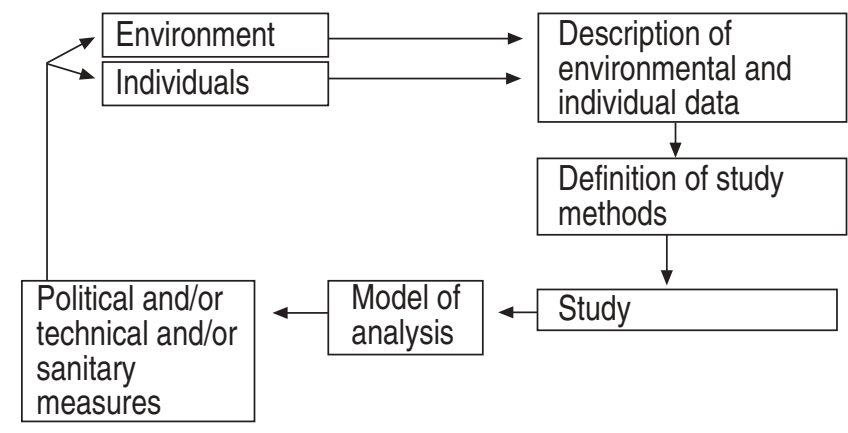

Fig. 1.- Model of a prospective study in environmental epidemiology.

Overall, a better understanding of pathophysiological mechanisms is needed, to enhance understanding of the observed excess morbidity and mortality. This can be accomplished by an interaction of epidemiological studies with human experimental chamber studies as well as relevant in vivo animal and in vitro models.

\section{Human experimental studies}

There is an obvious need for human experimental studies performed in individuals both with and without disease. Experimental chamber systems have long been in use for studies in healthy and asthmatic individuals, and for obvious reasons individuals with COPD are one of the major targets for future research. This is especially important given the quite pronounced pro-inflammatory events observed in bronchomucosal biopsies and bronchoalveolar lavage in healthy young adults following exposure to diesel engine exhaust. The findings of changes in antioxidant levels, enhanced expression of key cytokines in the bronchial epithelium, higher expression of endothelial adhesion molecules mediating cell traffic from the blood, as well as enhanced neutrophil, mast cell and lymphocytic infiltration offer possible links to the adverse health effects found in epidemiological studies [26, 27]. A thrombocytemia in the peripheral blood may correlate to the adverse cardiovascular endpoints met in epidemiological research. While local invasive investigations in the airways using a bronchoscope probably gives an unchallenged precision in tissue and cell sampling, the procedures are associated with a slightly increased risk of adverse events in elderly individuals with a number of diseases. The development of less invasive sampling techniques is therefore beneficial and has a very good potential. The use of induced sputum, measurements of exhaled nitric oxide and collection of frozen breath condensate has opened up new possibilities for noninvasively studying local airway responses in elderly and more fragile individuals $[28,29]$. It can be argued that the techniques may lack some sensitivity as compared with the more invasive techniques, but they are nevertheless to be taken very seriously as valuable research tools.

The cardiovascular system offers a number of important possibilities for identification and characterisation of air pollution-related events. Coagulation-related parameters, heart rate variability and arrhythmias are some of the more accessible endpoints to study. Recent observations have shown that patients with implanted defibrillating pacemakers experienced an increased rate of near-death events (with discharge due to ventricular fibrillation), with a lag time of a few days following air pollution episodes. Even though this suggests that arrythmogenic aspects may be important in certain individuals, it is not yet known whether the excess cardiac risk arises from electrical events or, as proposed by others, from alterations in blood coagulation, endothelial plaque disruption or other inflammatory events in the vascular walls. Therefore additional aspects of blood coagulation, arrhythmias, heart rate variability and arterial biology are important to assess.

\section{Animal studies}

Animal studies have sometimes been criticised for including exposure conditions, which are not relevant for humans, and species differences have sometimes been highlighted. While it should be kept in mind that there are a number of issues, which differ between man and rodents, the animal models do offer certain advantages. First of all, studies are easier and more practical to perform as compared to human exposure studies. Second, a complementary series of investigations clarifying patterns of responses are easier to do in animals. The identification of different strains of rats and mice, that have conditions mimicking diseases in humans, has provided valuable tools. For example, spontaneously hypertensive rats have shown to be more sensitive to air pollutants in comparison with other strains [30]. Additionally there are other animal models of different aspects of cardiovascular disease, which have been used to some extent in determining effects of the adverse ozone and particulate matter pollutants like diesel particles, residual oil fly ash and carbon black [31]. Lung disease models of asthma, bronchitis and COPD are well established and may be useful. To what extent the aging of rats, living only a few years, is relevant to the situation in the elderly human can be debated. This is an area that requires research in its own right. The use of genetically designed mice is a most valuable tool to clarify the role of different polymorphisms. Virtually every cytokine, inflammatory component and other molecule today can be studied in knock-out mice and in mice overexpressing the gene. The complementary use of these models with exposures to oxidative or particulate pollutants will be helpful to dissect the role of a wide range of mechanisms. For research in the elderly, the role of pro-inflammatory, and down regulating mechanisms may be of particular importance together with the oxidative-stress and free-radical responses.

\section{In vitro systems}

The use of in vitro cell systems is a valuable complementary model whereby a multitude of different cultured cells can be used in studies. Cell types can be of different origin, such as inflammatory cells and epithelial cells of different areas of the airways or vasculature. They can also be freshly obtained and cultured as primary cell cultures or explants. Following exposure and interventions with oxidative and particulate pollutants signal transduction, stepwise inflammatory events, counter-regulatory, free radical and oxidative aspects can be clarified. Of particular interest will be the use of cells originating from elderly people who are either healthy or having diseases which may render them particularly susceptible. The in vitro cell models are expected to become more complex in the future. Different cell layers and interstitial components will be co-cultured and far better resemble the airways than existing models. Such in vitro models are expected to result in a considerably more detailed understanding of the reactions in the airways.

Another interesting model to use would be epithelial lining fluid (ELF), that represents the first physical surface that inhaled pollutants interact with. As such, being rich in antioxidant and free-radical modulating substances, differences in responses between individuals with pre-existing 
susceptibility may be clarified further. Studies on ELF and related biological material from elderly and diseased animals and human individuals would also be of interest to study. The use of different chelating and binding molecules may be an effective way of dissecting the contribution of various metals, and oxidative and free-radical components involved in the interactions between air pollutants and the ELF.

\section{Conclusion}

Identification of the risk factors and mechanisms that underlie the epidemiology of adverse respiratory and cardiovascular morbidity and mortality in relationship to air pollutants in the elderly is of great importance from both the ethical and scientific perspective. Information on the source and physicochemical characteristics of particulate-matter pollution in relation to adverse reactions in the elderly may be of particular interest for European and worldwide air pollution policy decisions. By focusing on the elderly, who are the principal group at risk for cardiorespiratory mortality and morbidity, it may be possible to identify gaseous and particulate matter factors that can be specifically remedied, and which are practically and politically more achievable than current efforts to achieve a general reduction of air pollution levels without understanding what really matters.

\section{References}

1. Smith KR, Samet JM, Romieu I, Bruce N. Indoor air pollution in developing countries and acute lower respiratory infections in children. Thorax 2000; 55: 518-532.

2. Schindler C, Kunzli N, Bongard JP, et al. Short-term variation in air pollution and in average lung function among never-smokers. The Swiss Study on Air Pollution and Lung Diseases in Adults (SAPALDIA). Am J Respir Crit Care Med 2001; 163: 356-361.

3. Kunzli N, Kaiser R, Medina S, et al. Public-health impact of outdoor and traffic-related air pollution: a European assessment. Lancet 2000; 356: 795-801.

4. Brunekreef B. PEACE study. Eur Respir Rev 1998; 8: 52, 1-130.

5. Katsouyanni K, Zmirou D, Spix C, et al. Short-term effects of air pollution on health: a European approach using epidemiological time-series data. The APHEA project: background, objectives, design. Eur Respir J 1995; 8: 10301038 .

6. Murray CJ, Lopez AD. Alternative projections of mortality and disability by cause 1990-2020: Global Burden of Disease Study. Lancet 1997; 349: 1498-1504.

7. World Health Organization. Bronchial asthma. WHO Fact Sheet $\mathrm{N}^{\circ}$ 206. http://www.who.int/inf-fs/en/fact206.html. Date updated: January 2000; Date accessed: 3 February 2003.

8. Dockery DW, Pope CA 3rd, Xu X, et al. An association between air pollution and mortality in six U.S. cities. $N$ Engl J Med 1993; 329: 1753-1759.

9. Zeger SL, Dominici F, Samet J. Harvesting-resistant estimates of air pollution effects on mortality. Epidemiology 1999; 10: 171-175.

10. Hoek G, Brunekreef B, Fischer P, van Wijnen J. The association between air pollution and heart failure, arrhythmia, embolism, thrombosis, and other cardiovascular causes of death in a time series study. Epidemiology 2001; 12: 355357.

11. Rossi G, Vigotti MA, Zanobetti A, Repetto F, Gianelle V, Schwartz J. Air pollution and cause-specific mortality in Milan, Italy, 1980-1989. Arch Environ Health 1999; 54: 158164.
12. Eilat E, Mendlovic S, Doron A, Zakuth V, Spirer Z. Increased apoptosis in patients with major depression: A preliminary study. J Immunol 1999; 163: 533-534.

13. Cover H, Irwin M. Immunity and depression: insomnia, retardation, and reduction of natural killer cell activity. J Behav Med 1994; 17: 217-223.

14. Segal RA, Martonen TB, Lim CS, Shearer M. Computer simulations of particle deposition in the lungs of chronic obstructive pulmonary disease patients. Inhal Toxicol 2002; 14: 705-720.

15. Mudway IS, Kelly FJ. Ozone and the lung: a sensitive issue. Mol Aspects Med 2000; 21: 1-48.

16. Viegi G, Pedreschi M, Baldacci S, et al. Prevalence rates of respiratory symptoms and diseases in general population samples of North and Central Italy. Int J Tuberc Lung Dis 1999; 3: 1034-1042.

17. Viegi G, Pedreschi M, Pistelli F, et al. Prevalence of airways obstruction in a general population: European Respiratory Society vs American Thoracic Society definition. Chest 2000; 117: 5Suppl. 2, 339S-345S.

18. Lange P, Parner J, Vestbo J, Schnohr P, Jensen G. A 15-year follow-up study of ventilatory function in adults with asthma. N Engl J Med 1998; 339: 1194-1200.

19. Viegi G, Pedreschi M, Baldacci S, et al. Prevalence rates of respiratory symptoms and diseases in general population samples of North and Central Italy. Int J Tuberc Lung Dis 1999; 3: 1034-1042.

20. Viegi G, Pedreschi M, Pistelli F, et al. Prevalence of airways obstruction in a general population: European Respiratory Society vs American Thoracic Society definition. Chest 2000; 117: 5 Suppl. 2, 339S-345S.

21. Bakke P, Eide GE, Hanoa R, Gulsvik A. Occupational dust or gas exposure and prevalences of respiratory symptoms and asthma in a general population. Eur Respir $J$ 1991; 4 : 273-278.

22. Dow L, Coggon D, Osmond C, Holgate ST. A population survey of respiratory symptoms in the elderly. Eur Respir $J$ 1991; 4: 267-272.

23. McMichael AJ, Anderson HR, Brunekreef B, Cohen AJ. Inappropriate use of daily mortality analyses to estimate longer-term mortality effects of air pollution. Int J Epidemiol 1998; 27: 450-453.

24. Nordenhäll C, Pourazar J, Ledin MC, Levin JO, Sandström T, Ädelroth E. Diesel exhaust enhances airway responsiveness in asthmatic subjects. Eur Respir J 2001; 17: 909-915.

25. Sydbom A, Blomberg A, Parnia S, Stenfors N, Sandström T, Dahlen SE. Health effects of diesel exhaust emissions. Eur Respir J 2001; 17: 733-746.

26. Salvi SS, Nordenhäll C, Blomberg A, et al. Acute exposure to diesel exhaust increases IL-8 and GRO-alpha production in healthy human airways. Am J Respir Crit Care Med 2000; 161: 550-557.

27. Salvi S, Blomberg A, Rudell B, et al. Acute inflammatory responses in the airways and peripheral blood after shortterm exposure to diesel exhaust in healthy human volunteers. Am J Respir Crit Care Med 1999; 159: 702-709.

28. Montuschi P, Barnes PJ. Analysis of exhaled breath condensate for monitoring airway inflammation. Trends Pharmacol Sci 2002; 23: 232-237.

29. Nordenhäll C, Pourazar J, Ledin MC, Levin JO, Sandström T, Ädelroth E. Diesel exhaust enhances airway responsiveness in asthmatic subjects. Eur Respir $J$ 2001; 17: 909-915.

30. Kodavanti UP, Schladweiler MC, Ledbetter AD, et al. Temporal association between pulmonary and systemic effects of particulate matter in healthy and cardiovascular compromised rats. J Toxicol Environ Health 2002; 65: 15451569 .

31. Oberdorster G. Pulmonary effects of inhaled ultrafine particles. Int Arch Occup Environ Health 2001; 74: 1-8. 